Crime, Histoire \& Sociétés / Crime, History \& Societies

Vol. 2, n² | 1998

Histoire de la criminalité et de la justice pénale en Amérique latine / Criminal justice history in Latin America

\title{
Les juristes français et le régime de Vichy : à propos de deux ouvrages récents
}

René Lévy

URL : https://journals.openedition.org/chs/973

DOI : $10.4000 /$ chs. 973

ISSN : $1663-4837$

Éditeur

Librairie Droz

Édition imprimée

Date de publication : 1 juin 1998

Pagination : 111-119

ISBN : 2-600-00311-8

ISSN : $1422-0857$

\section{Référence électronique}

René Lévy, "Les juristes français et le régime de Vichy : à propos de deux ouvrages récents », Crime, Histoire \& Sociétés / Crime, History \& Societies [En ligne], Vol. 2, n² I 1998, mis en ligne le 03 avril 2009, consulté le 22 mars 2022. URL : http://journals.openedition.org/chs/973 ; DOI : https://doi.org/ $10.4000 /$ chs. 973 


\section{Les juristes français et le régime de Vichy: à propos de deux ouvrages récents'}

$\mathrm{W}$ eisberg (Richard H.), Vichy Law and the Holocaust in France (préface de Michael R. Marrus), New York, New York University Press (The Vidal Sassoon International Center for the Study of Antisemitism (SICSA), The Hebrew University of Jerusalem), 1996, xxiii + 447 p. (ISBN 0-8147-9302-9).

Badinter (Robert), Un antisémitisme ordinaire. Vichy et les avocats juifs (19401944), Paris, Fayard, 1997, 257 p. (ISBN 2-213-59802-9).

Les recherches sur la «collaboration» avec l'occupant allemand pendant la Seconde Guerre mondiale connaissent actuellement un développement considérable en France et cette question fait l'objet d'un débat public intense, alimenté par l'ultime vague des procès de certains des acteurs de cette période ${ }^{2}$. Après le procès de Klaus Barbie, qui avait permis d'éclairer la répression exercée directement par les nazis, et celui de Paul Touvier, qui avait mis l'accent sur la participation à cette entreprise des «collaborationnistes», ces Français qui s'étaient mis directement au service de l'occupant, c'est maintenant la collaboration d'État, où des fonctionnaires français ont participé à l'entreprise nazie sous l'autorité du gouvernement de Vichy, qui est au centre du procès de Maurice Papon. Après six mois d'audiences, il a finalement été condamné, en avril 1998, à 87ans, à dix ans de réclusion criminelle pour complicité de crimes contre l'humanité. Ce procès aurait normalement dû être précédé de celui d'un des principaux acteurs des événements en question, René Bousquet, ancien chef de la police de Vichy, assassiné par un déséquilibré avide de publicité quelques semaines avant sa comparution devant les juges. Blanchi à la Libération, il avait su se reconvertir dans les affaires et y avait fait une brillante carrière.

Même si Papon fut un protagoniste de moindre importance, sa mise en jugement illustre très bien le changement survenu en France, depuis 25 ans, dans la perception du régime de Vichy. Cet ancien secrétaire général de la préfecture de la Gironde et qui, à ce titre, organisa des convois de déportés Juifs, fut non seulement blanchi à la Libération pour services rendus à la Résistance, mais fit une grande carrière dans

1 Une version quelque peu différente de cet essai a été publicée en anglais sur $H$-Law/H-Net Humanities \& Social Sciences on Line (http ://www.h-net.msu.edu) en oct. 1997; je remercie Charles Zelden (Book review editor, H-Law) de m'avoir autorisé à en faire usage ici. La présente version a été complétée pour tenir compte de certains ouvrages plus récents.

2 Sur les débats suscités par le régime de Vichy, voir Rousso (1990); Conan, Rousso (1994); Azema, Bédarida (1992). On trouvera une bibliographie récente sur cette période dans: Farcy, Rousso (1993). 
l'administration préfectorale ${ }^{3}$, et fut même ministre au début des années 1970. C'est donc un notable de la Cinquième République qui se voit aujourd'hui jugé pour crimes contre l'humanité par une cour d'assises.

La question de la collaboration d'État est ainsi aujourd'hui en pleine lumière, mais il a fallu attendre l'accession à la présidence de Jacques Chirac, en 1995, pour voir reconnaître au plus haut niveau la responsabilité de la France dans les agissements de «l'autorité de fait dite 'gouvernement de l'État français'», comme la terminologie juridique officielle désigne le régime qui a dirigé le pays de 1940 à 1944 . Jusqu'alors, en effet, dans l'esprit des dirigeants successifs, de De Gaulle à Mitterrand, la République ne pouvait en aucune manière être comptable de régime de Vichy, son ennemi, dont l'inexistence juridique avait été proclamée par l'ordonnance du 8 août 1944. On en a longtemps tiré la conséquence que la République n'avait pas à commémorer la Grande rafle du Vel d'Hiv' de juillet 1942, effectuée par les policiers français, ni les autres méfaits antisémites de Vichy, bien qu'ils eussent été accomplis au nom de la France ${ }^{4}$.

Cette conception du régime de Vichy comme un «État fantoche» au service des nazis et dépourvu de toute légitimité propre, ne portait pas à examiner en détail son fonctionnement, ni à s'appesantir sur la continuité des institutions et du personnel administratif avant, pendant et après la guerre (que le cas Papon illustre clairement). Cette historiographie est aujourd'hui dépassée et le mérite en revient en grande partie à Robert Paxton et Michaël Marrus ${ }^{5}$, qui ont mis l'accent sur l'autonomie du régime de Pétain par rapport à l'Allemagne nazie et sur le caractère autochtone de son antisémitisme. C'est dans le prolongement de ce qui apparaît aujourd'hui comme le paradigme dominant que se situent les récentes publications qui portent sur le fonctionnement de ce régime, au sein desquelles les institutions juridiques et judiciaires font l'objet d'une attention particulière ${ }^{6}$.

C'est dans ce mouvement que se situe clairement l'ouvrage de R.H. Weisberg, qui vient compléter les travaux publiés par la revue Le genre humain ${ }^{7}$ et dont le sujet est également abordé, dans une perspective plus limitée, par le récent ouvrage de $\mathbf{R}$. Badinter sur l'élimination des avocats juifs du Barreau.

Le propos de Weisberg est le plus ambitieux des deux: alors que Badinter se limite au sort tragique d'une corporation particulière, qu'il retrace avec clarté et concision, Weisberg cherche à dresser un tableau complet de l'élaboration et de la mise en œuvre du droit antisémite, dans tous les domaines et en examinant aussi

3 Il fut notamment préfet de police de Paris, l'un des postes les plus importants dans l'administration française, à une époque particulièrement délicate et troublée, à la fin de la guerre d'Algérie. À ce titre, il porte une responsabilité dans le massacre que les policiers parisiens perpétrèrent le 17 octobre 1961 sur des manifestants algériens partisans de l'indépendance de l'Algérie; le nombre des victimes est aujourd'hui estimé à 200 , mais à l'époque on n'en reconnut officiellement que 7 , et aucune poursuite n'eut lieu (sur ces événements, voir Einaudi (1991), Levine (1985)). L'évocation de ces événements lors du récent procès Papon a suscité une nouvelle enquête officielle qui a révalué le nombre minimum de victimes à 32 .

4 Sur les inconséquences juridiques de cette conception, voir Rousseau (1994).

5 Paxton (1973) Marrus, Paxton (1981).

- Sur l'administration sous Vichy, l'ouvrage de référence est désormais celui de Baruch (1997). Sur le droit vichyste et les errements des juristes, il faut rappeler les articles fondamentaux de Danièle Lochak (1989, 1996); voir aussi le commentaire de Michel Troper (1989), ainsi que celui de Camy (1997).

7 Juger sous Vichy (1994); Le droit antisémite de Vichy (1996). 
bien l'action du gouvernement, des administrations, des tribunaux, des fonctionnaires, des magistrats que des autres professions judiciaires. Il nous livre un ouvrage foisonnant, qui défriche de nombreux terrains jusqu'ici peu explorés, mais qui aurait gagné à une construction plus rigoureuse ${ }^{8}$.

Le leitmotiv de l'ouvrage, divisé en dix chapitrès, est l'idée selon laquelle, sous l'angle juridique, la participation de Vichy à l'extermination des Juifs ne se ramène pas à l'œuvre d'une minorité de collaborateurs, mais a impliqué l'ensemble du système juridique français: après la défaite, ce dernier, resté intact dans l'ensemble, a absorbé sans difficulté les nouvelles mesures raciales et religieuses décidées par le régime sans la moindre pression allemande, et a appliqué toute sa compétence technique à rationaliser la prétendue différence irréductible des Juifs sur laquelle elles reposaient. Le propos de l'auteur est, explicitement, de dresser un réquisitoire contre les juristes français, coupables de n'avoir exercé leur talent qu'à sens unique, c'està-dire sans remettre en cause la légitimité du droit nouveau qu'ils s'appliquaient à élaborer, analyser ou mettre en cuvre, alors même que dans d'autres domaines, ils se montraient beaucoup plus combatifs pour préserver les principes juridiques républicains?.

C'est le cas par exemple, dans le procès de Riom (février-avril 1942), destiné, aux yeux de Pétain, à mettre en lumière les responsabilités des principaux dirigeants de la IIIe République, et par lequel Weisberg a choisi d'ouvrir son livre. Ce procès, et en particulier le sort fait à Léon Blum, lui paraît en effet exemplaire des contradictions du régime et des ambiguïtés des juristes: il vise à l'expulsion symbolique hors de la communauté française de celui (Blum) qui représente tout ce que honnit le régime (démocratie parlementaire, socialisme, judaïsme). Mais le procès, qui se tient devant une juridiction d'exception, reste empreint de légalisme et l'antisémitisme n'y fait qu'occasionnellement surface (p. 15). Surtout, il met en lumière le fait qu'il était possible, même dans ces circonstances, de pratiquer une défense politique, sans concession à l'idéologie du régime, et sans s'exposer pour autant à des représailles. Et lorsque, excédé par la tournure du procès, Pétain tente de court-circuiter le tribunal, on voit intervenir énergiquement le bâtonnier de Paris, Jacques Charpentier, qui avait pourtant accepté sans sourciller les mesures antisémites touchant le Barreau (p. 22).

Les chapitres suivants sont consacrés à l'exposé de la législation antisémite (chapitre 2), et à leur mise en œuvre à l'encontre des avocats, magistrats et autres professionnels du droit juifs; Weisberg expose en particulier les divergences d'interprétation entre le ministère de la Justice, les tribunaux et le Commissariat général aux questions juives (CGQJ) (chapitre 3). Le chapitre 4 est consacré à la personnalité de Joseph Barthélemy, deuxième ministre de la Justice de Vichy que son passé de juriste libéral sous la III ${ }^{c}$ République ne paraissait pas prédisposer à cette fonction.

8 Signalons ici, pour ne plus y revenir, quelques défauts mineurs qu'une relecture attentive eût pu prévenir: Dominique Gros est référencé tantôt comme Gros (D.), et tantôt comme Dominique-Gros (cf. p.55, n.61 et p.432); le nom de Henri Amouroux est transcrit en Amoureux (p. 371 et 431). L'auteur ne recule pas, en plusieurs endroits, devant certains clichés agaçants, tels que «almost germanic sense of precision» (p. 304) ou «a gritty Gallic perspicacity» (p. 313).

- Ce point de vue est partagé par Baruch (1997,p. 579 et passim), qui observe que les administrations surent trouver, pour protéger leurs attributions contre les empiètements du régime et les tendances «collaborationnistes», des marges de manœuvre juridiques qu'elles ne surent pas se ménager pour protéger les principes républicains ni, en particulier, pour lutter contre les mécanismes d'exclusion politique ou raciale. 
Les chapitres 5 à 7 reviennent en détail sur les difficultés techniques de la mise en œuvre de la législation antisémite: le chapitre 5 s'appesantit sur la charge de la preuve en matière d'établissement de la judéité et sur les divergences entre le CGQJ et les tribunaux quant à la compétence des différents ordres de juridiction; le chapitre 6 traite des difficultés dans la mise en œuvre des critères légaux de judéité; le chapitre 7 examine les questions patrimoniales suscitées par la législation antisémite et notamment les questions liées à l'aryanisation des biens.

Ces chapitres consacrés à l'application du droit antisémite, qui soulèvent des questions techniques complexes que l'auteur expose clairement, sont particulièrement intéressants, parce qu'ils reposent sur l'examen des dossiers d'archives, et non pas seulement sur la jurisprudence publiée, comme c'est le cas pour les contributions correspondantes de l'ouvrage sur Le droit antisémite de Vichy. Ils montrent aussi l'intense activité juridique et juridictionnelle suscitée par ce nouveau droit et le profond juridisme de celle-ci: exclusion et spoliation, certes, mais dans les formes! Ils mettent également en lumière les luttes d'influence entre les différentes branches de l'État-CGQJ, tribunaux judiciaires ou administratifs - pour la maîtrise de ce droit. La confrontation de ces deux sources devrait faire l'objet d'études spécifiques ultérieures, qui permettraient, dans chaque domaine, de confronter la réalité du contentieux jugé avec la fraction de celui-ci qui, par des circuits complexes, fait l'objet d'une publication et d'un commentaire savant, qui rétroagissent nécessairement sur la manière de juger.

Le chapitre 8 étudie en détail la situation des avocats: en ce qui concerne l'éviction des avocats juifs, et le rôle du Barreau et de ses dirigeants eux-mêmes dans cette entreprise, l'analyse de Weisberg est corroborée par celle de Badinter, ce qui n'est guère étonnant, leurs sources étant identiques ${ }^{10}$, mais là encore, Weisberg a fait œuvre de pionnier. C'est également le cas en ce qui concerne la troisième partie de ce chapitre, consacrée au traitement du contentieux relatif aux lois antisémites par les avocats non juifs, qui mériterait certainement d'être approfondi sur un corpus plus important ${ }^{11}$.

Le chapitre 9, un peu fourre-tout, traite d'abord des vaines tentatives du CGQJ pour obtenir, en 1943, une extension des dénaturalisations, qui aurait permis de nouvelles déportations; une deuxième section traite de diverses réformes judiciaires, abouties ou non, et en particulier des «sections spéciales», questions qui n'ont qu'un rapport marginal avec le sujet de l'ouvrage; la troisième section évoque le cas particulier des enfants adultérins, protégés d'une recherche en judéité par l'existence, dans le droit français de l'époque, d'une prohibition générale de la recherche de paternité.

Chacun de ces chapitres est intéressant en soi, mais leur assemblage fait davantage songer à une collection d'articles réunis à la hâte qu'à un ouvrage rationnellement construit, et ce défaut obscurcit le propos. Les mêmes questions sont abordées à plusieurs reprises dans des chapitres parfois éloignés, sans raison apparente, de sorte que redites et renvois abondent et qu'il est difficile de prendre une vue d'ensemble des questions traitées. La législation antisémite est exposée au chapitre 2 ,

10 Badinter est parfois plus précis que Weisberg sur des événements particuliers: par exemple au sujet de la démarche effectuée par certains avocats antisémites auprès des Allemands, pour obtenir la libération de plusieurs avocats juifs éminents internés à Drancy (Cpr. Badinter, 1997, 145 sq., Weisberg, 1996, p. 92); inversement, Weisberg révèle les noms des intéressés, que Badinter omet.

"Une partie de ce chapitre, consacrée à l'analyse des archives du célèbre avocat Maurice Garçon, a été publiée dans Le droit antisémite de Vichy. 
mais la loi xénophobe de septembre 1940 qui la précède n'est évoquée qu'au chapitre 8. Les questions relatives au statut personnel sont ainsi abordées aux chapitres $2,5,6$ et 9 , alors qu'il aurait été plus judicieux de les regrouper dans une même par$t^{1} \mathrm{e}^{12}$. Les questions patrimoniales sont abordées aux chapitre 6 (D), 7 et 8 (C). Le sort des professions judiciaires, et en particulier le numerus clausus frappant les avocats juifs, est traité d'abord au chapitre 3, puis au chapitre 8. Quant aux aspects idéologiques de la question, et notamment l'importance de l'antijudaïsme chrétien, ils sont traités aux chapitres 1,4 et 10 .

La lecture de l'ouvrage suppose donc une bonne connaissance préalable de l'histoire de la période et également de l'organisation des tribunaux et des diverses professions judiciaires, toutes questions qui eussent peut-être mérité un bref exposé liminaire.

Sur le fond, le constat d'ensemble de Weisberg ne s'éloigne guère de celui que formulait Lochak dans son article de 1989: en se conduisant en purs techniciens du droit, fût-il antisémite, la majorité des juristes ont contribué à banaliser, déréaliser et finalement légitimer la discrimination, l'exclusion et la spoliation. C'est dans l'explication de cette attitude que Weisberg se sépare de Lochak, qui y voyait une conséquence du positivisme juridique dont les juristes étaient imprégnés, et il propose, dans le chapitre 10, une autre explication. Sa thèse, sommairement résumée, est la suivante:

- l'existence de lois antijuives ne suffit pas à expliquer le comportement de l'establishment juridique vichyssois à l'égard des Juifs, de telles lois étant restées largement inappliquées en Italie, par exemple;

- le facteur déterminant est ce que l'auteur appelle «l'herméneutique vichyssoise » (Vichy hermeneutics), qui a rapidement imprégné l'ensemble de la pratique juridique et a conduit à exclure les Juifs de la protection juridique accordée aux «vrais Français». Elle consiste en une grille de lecture des situations juridiques qui se caractérise par une interprétation souple de ce qui subsiste des principes constitutionnels anciens (par ex. de la notion d'égalité), combinée à une interprétation stricte et purement technique des lois nouvelles (p. 389).

- Il y a combinaison d'une relecture des principes constitutionnels (selon une technique qui évoque, d'après l'auteur, les thèses déconstructionnistes d'inspiration française aujourd'hui en faveur aux États-Unis (p. 388), mais surtout la relecture chrétienne originelle de l'Ancien Testament) avec un cartésianisme myope qui pousse la législation antisémite jusqu'à ses conséquences les plus extrêmes, au lieu d'utiliser les ressources de la technique et de la rhétorique juridiques contre cette dernière (p. 389).

- L'auteur estime, en définitive, que c'est un mode particulier de raisonnement catholique français qui explique la perméabilité aux idées de Vichy d'une culture juridique qui leur était en principe réfractaire (p. 389). C'est pourquoi les commentaires juridiques des lois antisémites adoptent une perspective beaucoup plus religieuse que raciale, ce qui conduit l'auteur à parler de lois religieuses plutôt que de lois raciales, à propos du statut des Juifs. Les juristes français seraient

12 Au chapitre 2, les p. 66 sq. traitent des mêmes questions que les sections A et B du chapitre $5 ;$ La question des enfants adultérins, abordée p. 150-152, ressurgit p. 380-385. 
ainsi davantage influencés par le vieil antijudaïsme chrétien que par le racisme nazi : leur manière de lire le droit s'apparenterait à la lecture catholique traditionnelle de l'Ancien Testament, lecture qui procèderait à l'expulsion du Juif hors du texte biblique.

Que la France ait possédé sa propre tradition antisémite, distincte du racisme biologique nazi, et fortement teintée d'antijudaïsme catholique ne fait pas de doute; Xavier Vallat ne se gênait d'ailleurs pas pour le faire remarquer à Dannecker, ce qui causa son éviction du CGQJ comme le signale Badinter (p. 172). Que l'Église catholique ait perçu l'avènement du nouveau régime comme «une divine surprise», est également bien connu. Mais l'attribution, par Weisberg, de cette faillite collective des juristes, que nul ne conteste plus - et qui n'empêcha d'ailleurs pas des comportements individuels honorables, sur lesquels l'auteur insiste à de multiples reprises - à la prégnance de la tradition catholique anti-judaïque dans la mentalité des juristes n'emporte pas la conviction.

Sans entrer ici dans la discussion de l'interprétation que fait Weisberg de l'exégèse catholique du Pentateuque, qui dépasse nos compétences, on remarquera que son parallèle repose en partie sur un contresens: si l'on admet que dans le premier cas, celui de l'exégèse catholique, il s'agit d'exclure (read out) le Juif de la Bible, tel n'est pas le cas dans le droit de Vichy, bien au contraire! Comme le montre la frénésie législative du régime (168 textes relatifs aux Juifs en 4 ans $)^{13}$, il s'agit d'injecter le Juif, si l'on ose dire, dans toutes les branches d'un droit qui, dans la tradition républicaine, se refusait absolument à en connaître. Dorénavant, le mot «juif » surgit en quelque sorte de chaque article de loi: le droit ne peut plus être interprété en ignorant cette catégorie juridique nouvelle, mais doit être entièrement relu à sa lumière. D'où la multiplication des contentieux, parfois les plus absurdes, dans des domaines imprévus, comme par exemple la discussion de la judéité de telle ou telle secte ou bien des enfants dont la filiation est inconnue. D'où également les efforts intenses des juristes pour réintégrer les problèmes nouveaux dans des cadres juridiques connus, occultant par là-même le caractère odieux et les conséquences tragiques de ce droit ${ }^{14}$.

On peut, du reste, s'interroger sur les voies par lesquelles cette tradition catholique aurait pénétré la collectivité des juristes, formés dans un régime politique marqué par un conflit persistant avec l'Église. À tout le moins faudrait-il faire des distinctions entre les différentes catégories de juristes, que Weisberg tend à confondre sous l'appellation de «lawyer». Les avocats, les magistrats, les juristes des administrations, les membres du Conseil d'État ou les universitaires, n'ont ni les mêmes origines sociales, ni les mêmes intérêts, ni les mêmes traditions professionnelles (et en particulier le rapport à l'État), ni, vraisemblablement, les mêmes idées, à supposer même que ces catégories professionnelles fussent homogènes. Il manque, en somme, à cet ouvrage de s'adosser à une sociologie des professions juridiques et judiciaires.

Peut-être n'est-il pas besoin de remonter à une supposée tradition catholique pour trouver les raisons de l'indifférence des juristes: la période de l'immédiat avantguerre avait largement préparé le terrain, et les esprits, en matière de xénophobie et d'antisémitisme. Curieusement, s'éloignant en cela de Marrus et Paxton, qui insis-

13 Gros (1996, p.41, n.37).

14 Lochak (1989, p.260-265). 
taient au contraire sur la profonde continuité entre les années 1930 et Vichy ${ }^{15}$, Weisberg ne fait pratiquement pas référence à cette période: il ne mentionne même pas la loi du 19 juillet 1934, adoptée sans débat et par laquelle le Barreau obtint la fermeture de la profession d'avocat aux étrangers et aux Français naturalisés depuis moins de dix ans ${ }^{16}$. Selon Badinter, le Barreau de Paris, le plus important de France, était traditionnellement antisémite et veillait à ce que les avocats juifs ne pussent accéder aux honneurs et aux responsabilités en son sein ${ }^{17}$.

Dans le même ordre d'idées, le jugement sur la manière dont les membres de chacune de ces catégories ont ou non participé à l'entreprise vichyste devrait tenir compte des contraintes spécifiques à chacune: les magistrats doivent être évalués à l'aune des jugements rendus, dès lors qu'ils n'ont pas démissionné et sont tenus de juger; les avocats ne sauraient se voir reprocher d'avoir joué au plus fin avec le droit en vigueur, dès lors que leurs clients persécutés le demandaient; quant aux professeurs de droit, rien ne les contraignait à la connivence avec un tel droit ${ }^{18}$. Il est dommage, de ce point de vue, que l'auteur ne se réfere pas à la typologie élaborée par Burrin qui distingue différentes formes de collaboration, selon qu'elles sont guidées par la raison d'Etat, le souci d'accommodement ou l'engagement. Baruch, appliquant ces distinctions à la collaboration administrative, a ainsi fort bien analysé la complexité du phénomène où s'entremêlent, sans qu'il soit toujours possible de les distinguer, considérations idéologiques, volonté de revanche, ambitions personnelles, conformisme et sens de l'État fréquemment fourvoyé en «syndrome de la rivière Kwaï» ${ }^{19}$. Les choses ne sont du reste pas moins complexes lorsqu'il s'agit d'analyser les différentes formes d'opposition à la collaboration, qui vont de la désobéissance ponctuelle à la résistance active, en passant par la dissidence, selon des modalités souvent très ambiguës où ont leur part, dans des proportions qui varient à mesure que la défaite allemande paraît plus probable, patriotisme sincère, esprit de corps, et opportunisme, comme l'a également montré Baruch ${ }^{20}$. L'importance de ces nuances se mesure à ce qu'elles permettent d'établir une périodisation fine des événements et des attitudes, que la construction de l'ouvrage de Weisberg ne permet pas de percevoir.

En définitive, la question de la participation des juristes au régime de Vichy ne se pose sans doute pas très différemment de celle des autres catégories de la population, dont la majorité, au moins dans les premiers temps, ne regretta pas la III ${ }^{\mathrm{e}}$ République et ne se formalisa pas de la législation antisémite. Dans la «Patrie des droits de l'Homme », on aurait assurément espéré mieux, et la déception rétrospective que suscite cette médiocre réalité chez ceux qui ont, comme Weisberg ou Badinter, voué leur vie au droit, est clairement perceptible dans leurs ouvrages.

is Marrus, Paxton (1981, p. 45). Sur l'attitude des professeurs de droit, voir l'étude minutieuse et impitoyable de Gros (1993) au sujet des manuels de droit publiés pendant l'Occupation.

16 Le même texte visait d'ailleurs l'ensemble de la fonction publique et des offices ministériels: LavalReviglio (1996). Des mesures restrictives du même type touchaient l'exercice de la médecine, ainsi que le commerce et l'artisanat. Voir aussi Bonnet (1976).

17 Badinter (1997, p. 20-21), qui indique également que le Conseil de l'ordre des avocats parisiens appliqua la loi de 1934 rétroactivement (p. 29).

18 Sur ce point, Lochak (1994); Lochak (1996, en particulier n. 25, p. 460). Sur les magistrats v. aussi (1996).

19 Burrin (1995); Baruch (1997, p. 366 s.).

20 Baruch (1997, p. 427 s.). 
Ces remarques n'enlèvent rien à l'intérêt de ces derniers qui constituent un apport substantiel et stimulant à un domaine aujourd'hui en plein développement.

\author{
René Lévy \\ CESDIP-CNRS \\ Immeuble Edison \\ 43, bd Vauban \\ F-78280 Guyancourt \\ rlevy@ext.jussieu.fr
}

\title{
RÉFÉRENCES
}

Azema, J.P., Bédarida, F., (Dir.), Vichy et les Français, Paris, Fayard, 1992.

Bancaud, A., La magistrature et la répression politique de Vichy ou l'histoire d'un demiéchec, Droit et Société, 1996, 34, p. 557-574.

Baruch, M.O., Servir l'État français. L'administration en France de 1940 à 1944, Paris, Fayard 1997.

Bonnet (J.-Ch.), Les pouvoirs publics français et l'immigration dans l'entre-deux-guerres, Lyon, Centre d'histoire économique et sociale de la région lyonnaise, 1976.

Burrin, Ph., La France à l'heure allemande, 1940-1944, Paris, Seuil, 1995 ( $2^{c}$ éd. Coll. Points, 1997).

Camy, O., Le positivisme comme moindre mal ? Réflexions sur l'attitude des juristes français face au droit antisémite de Vichy, Revue interdisciplinaire d'études juridiques, 1997, 39, p. $1-25$.

Conan, E., Rousso, H., Vichy, un passé qui ne passe pas, Paris, Fayard, 1994.

Einaudi, J.L., La bataille de Paris, 17 octobre 1961, Paris, Seuil, 1991.

Farcy, J.C., Rousso, H., Justice, répression et persécution en France (fin des années 1930début des années 1950). Essai bibliographique, Les Cahiers de l'lHTP, 1993, $\mathrm{n}^{\circ} 24$.

Gros, D., Le «Statut des Juifs» et les manuels en usage dans les facultés de droit (19401944): de la description à la légitimation, in Braud, Ph., (Dir.), La violence politique dans les démocraties européennes occidentales, Paris, L'Harmattan, 1993, p. 139-172.

Gros, D., Peut-on parler d'un droit antisémite?, in Le droit antisémite de Vichy, Paris, Seuil (Le genre humain), 1996, p. 13-44.

Juger sous Vichy, Paris, Seuil (Le genre humain), 1994.

Laval-Reviglio, M.C., Parlementaires xénophobes et antisémites sous la IIIe République, in Le droit antisémite de Vichy, Paris, Seuil (Le genre humain), 1996, p. 85-114.

Le droit antisémite de Vichy, Paris, Seuil (Le genre humain), 1996.

Levine, M., Les ratonnades d'octobre, un meurtre collectif à Paris en 1961, Paris, Ramsay, 1985.

Lochak, D., Le juge doit-il appliquer une loi inique? in Juger sous Vichy, Paris, Seuil (Le genre humain), 1994, p. 29-40.

Lochak, D., La doctrine sous Vichy ou les mésaventures du positivisme, in Les usages sociaux du droit, Paris, PUF, 1989, p. 252-285.

Lochak, D., Ecrire, se taire... Réflexions sur l'attitude de la doctrine française, in Le droit antisémite de Vichy, Paris, Seuil (Le genre humain), 1996, p. 432-462.

Marrus, M.R., Paxton, R.O., Vichy et les Juifs, Paris, Calmann-Lévy, 1981 (1 $1^{\text {re }}$ éd.: Vichy France and the Jews, New York, Basic Books, 1981). 
Paxton, R.O, La France de Vichy 1940-1944, Paris, Seuil, 1973 (1 $1^{\text {tre }}$ éd. :Vichy France, Old Guard and New Order, 1940-1944, New York, Columbia U.P., 1972).

Rousseau, D., Vichy a-t-il existé?, in Juger sous Vichy, Paris, Seuil (Le genre humain), 1994, p. 97-106.

Rousso, H., Le syndrôme de Vichy de 1944 à nos jours, Paris, Seuil 1990 ( $1^{\text {tre }}$ éd. 1987; trad. anglaise: The Vichy syndrome: History and Memory in France since 1944, Cambridge, Mass., Harvard U.P., 1991).

Troper, M., La doctrine et le positivisme (à propos d'un article de Daniele Lochak), in Les usages sociaux du droit, Paris, PUF, 1989, p. 286-292. 